\title{
Fundamentals of oxygen delignification. Part II. Functional group formation/elimination in residual kraft lignin
}

\author{
Faraj Asgari and Dimitris S. Argyropoulos
}

\begin{abstract}
The complex interaction of softwood residual kraft lignin with oxygen at elevated temperatures, pressures, and $\mathrm{pH}$ 's was investigated by isolating and systematically oxidizing the lignin, as a function of time and temperature. The isolation of the oxidized lignin was carried out using a multistep procedure developed specifically to recover all species. Detailed quantitative ${ }^{31} \mathrm{P}$ NMR measurements provided, for the first time, three-dimensional plots describing the formation and (or) elimination of the various functional groups. Oxidative demethoxylation reactions were found to induce the formation of catechols, which are, most likely, reactive intermediates of the oxidation. The rate of carboxylic acid formation and guaiacyl phenol elimination was found to follow two distinct phases: a rapid initial phase followed by a slower phase. More specifically, the rate of carboxylic acid group formation was found to dramatically increase as the reaction temperature increased, analogous to the profiles obtained for the elimination of guaiacyl phenolic units. At reaction temperatures typical of conventional commercial oxygen delignification installations $\left(80-100^{\circ} \mathrm{C}\right)$, only minor oxidation was found to occur within the lignin. At these temperatures, increasing the reaction time did not significantly aid the formation of carboxylic acid groups on lignin despite the idealized twophase homogeneous conditions. The technological ramifications of these data imply that a three-phase oxygen delignification system, operating at temperatures below $100^{\circ} \mathrm{C}$, causes only minor oxidative changes to the structure of the lignin in the fibre. It was also possible to isolate the effect of alkali and temperature on the structure of residual kraft lignin through experiments in which pressurized oxygen was replaced by nitrogen. These data revealed a concealed set of alkali-induced condensation and fragmentation reactions.
\end{abstract}

Key words: bleaching, catechols, carboxylic acids, delignification, guaiacyl, kinetics, oxygen, phenols, phosphorus, nuclear magnetic resonance (nmr), residual kraft lignin.

Résumé : En procédant à son isolement et son oxydation systématique en fonction du temps et de la pression, on a étudié l'interaction complexe de la lignine kraft résiduelle de bois mou avec l'oxygène à des températures, pressions et pH élevés. On a réalisé l'isolation de la lignine oxydée en appliquant une méthode en plusieurs étapes développées spécifiquement pour récupérer toutes les espèces. Des mesures quantitatives détaillées de $\mathrm{RMN}$ du ${ }^{31} \mathrm{P}$ ont permis d'obtenir pour la première fois des courbes tridimensionnelles décrivant la formation et (ou) l'élimination de divers groupes fonctionnels. On a trouvé que les réactions de déméthoxylation oxydante induisent la formation de catéchols qui sont vraisemblablement des intermédiaires réactifs dans l'oxydation. On a trouvé que la vitesse de formation de l'acide carboxylique et d'élimination de guaïacol phénolique suit deux phases distinctes : une phase initiale rapide suivie d'une phase plus lente. D'une façon plus spécifique, on a trouvé que la vitesse de formation d'acide carboxylique augmente d'une façon dramatique lorsqu'on élève la température; on a obtenu des profils analogues pour l'élimination des unités guaiacyles phénoliques. À des températures réactionnelles semblables à celles utilisées dans les installations commerciales conventionnelles de délignification par l'oxygène $\left(80-100^{\circ} \mathrm{C}\right)$, on observe peu d'oxydation de la lignine. À ces températures, une augmentation du temps de réaction n'aide pas d'une façon significative à la formation d'acides carboxyliques sur la lignine malgré les conditions homogènes en deux phases idéalisées. Les ramifications technologiques de ces données impliquent qu'un système de délignification à l'oxygène en trois phases, à des températures inférieures à $100^{\circ} \mathrm{C}$, ne provoque que des changements oxydants mineurs de la lignine de la fibre. Il a aussi été possible d'isoler l'effet des métaux alcalins et de la température sur la structure de la lignine kraft résiduelle en procédant à des expériences dans lesquelles l'oxygène sous pression est remplacé par de l'azote. Ces données révèlent certaines conditions induisent des réactions de condensation et de fragmentation.

Mots clés : blanchiment, catéchols, acides carboxyliques, délignification, guaiacyle, cinétique, oxygène, phénols, phosphore, résonance magnétique nucléaire, lignine kraft résiduelle.

[Traduit par la Rédaction]

Received June 16, 1998.

F. Asgari and D.S. Argyropoulos. ${ }^{1}$ PAPRICAN and Department of Chemistry, McGill University, Pulp \& Paper Research Centre, 3420 University Street, Montreal, QC H3A 2A7, Canada.

${ }^{1}$ Author to whom correspondence may be addressed. Telephone: (514) 398-6178. Fax: (514) 398-8254.

E-mail: dimitria@shared1.lan.mcgill.ca 


\section{Introduction}

Regulatory pressures, motivated by concerns regarding the toxicity of pulp and paper mill effluents containing chlorinated organic matter, have driven this industry to consider and (or) implement new delignification and bleaching technologies. In this context, the use of oxygen delignification is becoming of paramount global importance. The environmental, technical, and economic benefits of oxygen delignification include lower chemical requirements in the subsequent bleaching sequence, higher brightnesses with an equivalent amount of chemicals, lower amounts of rejects, and lower water consumption due to the greater recycling potential of oxygen stage effluent $(1,2)$. However, an oxygen delignification stage allows for limited delignification $(3,4)$. Beyond approximately $50 \%$ delignification, severe cellulose depolymerization takes place, causing deterioration of pulp viscosity and strength characteristics. This lack of selectivity is the main drawback of oxygen delignification (5).

The fundamental interactions of oxygen in aqueous alkali with various lignin model compounds have been the subject of various structural and kinetic enquiries (6-12). However, it should be noted that the conclusions of such studies cannot fully describe the events that occur when oxygen interacts with lignin macromolecules. The polymeric and crosslinked nature of lignin induces physical constraints that make it significantly less reactive toward oxygen than model compounds (12). It is likely that the multitude of branching and cross-linking variations that occur within lignin may cause significant accessibility variations of reactive moieties toward even the same functional groups.

Moreover, during oxygen delignification, molecular oxygen can be transformed into a variety of species possessing different reactivities, with different lignin functional groups adding to the complexity of the process. In actual fact, the stepwise reduction of molecular oxygen can proceed via either four one-electron transfer steps or two two-electron transfer steps (Scheme 1) (9). In the course of these transformations some extremely reactive species are formed, including hydroperoxy radicals, hydroxy radicals, and hydrogen peroxide (13).

Scheme 1. The stepwise reduction of molecular oxygen (biradical) via four one-electron transfer steps and two twoelectron transfer steps (9).

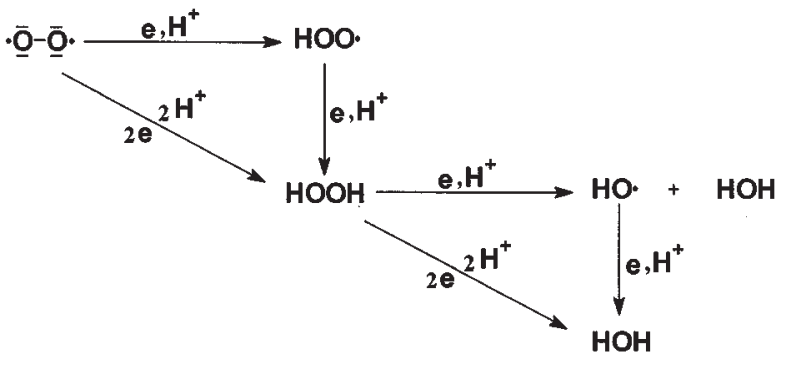

The reactions in Scheme 2, between the species produced during the stepwise reduction of oxygen depicted in Scheme 1, are also possible, both in the presence and in the absence of metal ions (14).
Scheme 2. The reactions that may occur between the species produced during the stepwise reduction of biradical oxygen in the presence and absence of metal ions (14).

$$
\begin{aligned}
& \text {. } \underline{\mathrm{O}}-\underline{\mathrm{Q}} \cdot \rightleftharpoons \mathrm{O}_{2}^{-} \\
& \mathrm{HOO}^{\circ}+\mathrm{O}_{2}^{-} \longrightarrow \cdot \underline{\overline{\mathrm{O}}}-\underline{\mathrm{o}} \cdot+\mathrm{HOO}^{-} \\
& \mathrm{HOOH}+\mathrm{HOO}^{-} \longrightarrow \mathrm{O}_{2}^{-}+\mathrm{HO}^{\circ}+\mathrm{HOH} \\
& \mathrm{HO}^{\circ}+\mathrm{O}_{2}^{-} \longrightarrow \cdot \underline{\overline{\mathrm{O}}}-\underline{\mathrm{o}} \cdot+\mathrm{HO}^{-} \\
& \mathrm{O}_{2}^{-}+\mathrm{HOOH} \stackrel{\text { Metal ions }}{\longrightarrow} \mathrm{HO}^{-}+\mathrm{HO}^{\circ}+\mathrm{O}_{2} \\
& \mathrm{HOOH}+\mathrm{HOO}^{-} \stackrel{\text { Metal ions }}{\longrightarrow} \mathrm{O}_{2}^{-}+\mathrm{HO}^{\circ}+\mathrm{HOH}
\end{aligned}
$$

The chemistry depicted in Schemes 1 and 2 clearly indicates that hydrogen peroxide is inevitably present under conditions of oxygen delignification. Therefore, both oxygen and hydrogen peroxide may cooperatively participate in defining the delignification chemistry of such systems. Gierer has classified hydroxyl radicals and the oxygen biradical as electrophilic species, while hydroperoxide anions and superoxide anion radicals are classified as nucleophilic species $(14,15)$. Consequently, the electrophiles will react with the

Scheme 3. The centres of high and low electron densities on the $\mathrm{C}_{9}$ unit of lignin. These are the respective sites for attack by electrophiles or nucleophiles.<smiles></smiles>

Center of high electron density<smiles>CC=CC=c1[c+]c(OC)c(=O)cc1</smiles>

Center of low electron density centres of high electron density in lignin, while the nucleophiles will react with the centres of low electron density as shown in Scheme 3.

Numerous studies have also demonstrated that the phenolic units of lignin are amongst the most significant reaction sites for oxygen delignification (11). The primary interaction of alkali with these structures yields anions that are candidate structures for attack by oxygen and its multitude of coexisting species (16).

In an effort to provide an additional perspective on the complex interaction of kraft lignin with oxygen in alkaline media at elevated temperatures and pressures, we isolated residual softwood kraft lignin and systematically oxidized it as a function of time and temperature. This was carried out to bridge the existing knowledge gap between model compounds and actual residual kraft lignin. Detailed quantitative ${ }^{31} \mathrm{P}$ NMR measurements on the oxidized lignins provided, for the first time, the actual kinetics of formation and (or) elimination of guaiacyl phenols, carboxylic acids, catecholic phenols, and aliphatic alcohols present in residual kraft lignin. 
Scheme 4. Schematic representation of the recovery method developed to quantitatively isolate lignin and its fragments after oxidation with oxygen at elevated temperatures and $\mathrm{pH}$ 's.

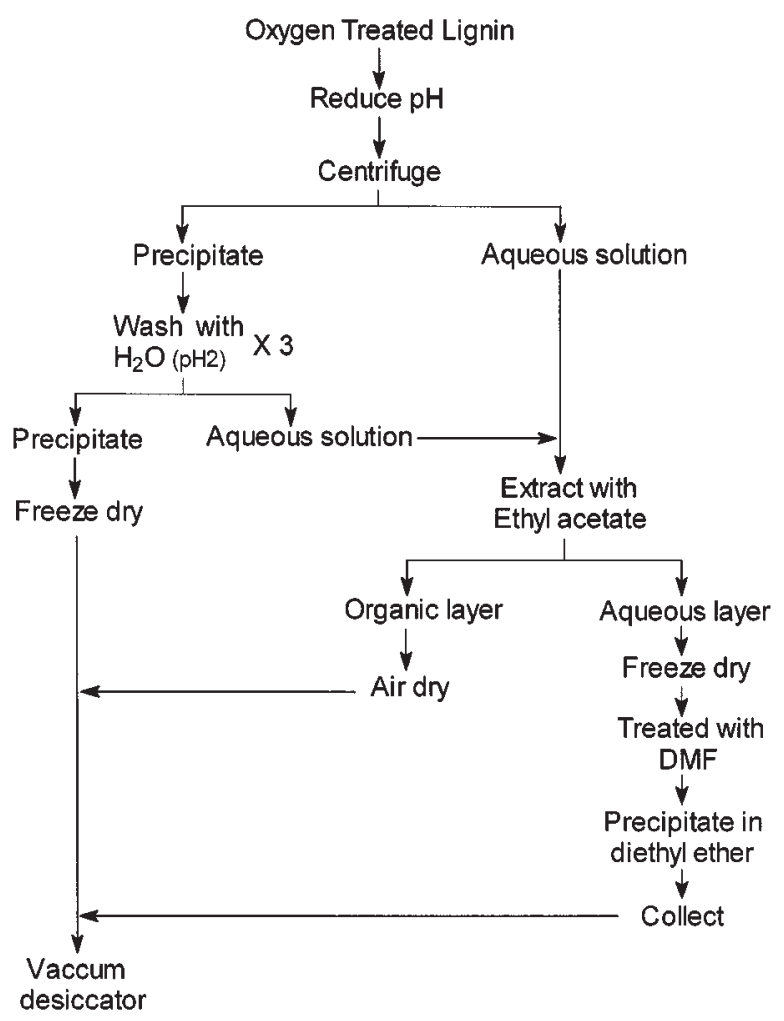

\section{Experimental}

\section{Isolation of residual kraft lignin}

A black spruce (Picea mariana) kraft pulp (kappa number 30.9) was freed from metal ions by stirring the pulp in a DTPA solution $\left(60^{\circ} \mathrm{C}, 1 \mathrm{~h}\right)$ and, after a washing step, the pulp was treated in an acid solution $\left(\mathrm{H}_{2} \mathrm{SO}_{4}\right.$ at $\mathrm{pH} 3$, room temperature, $2 \mathrm{~h}$ ), followed by thorough washing with deionized water. The residual lignin was then isolated from the pulp in several batches by using a slightly modified acidolysis procedure described elsewhere (17). The residual lignin samples were thoroughly homogenized to ensure sample uniformity. The yield of the isolation procedure varied between 35 and $45 \%$ while its purity was confirmed by UV and Klasson lignin content measurements.

\section{Oxidation of residual kraft lignin}

The residual kraft lignin was oxidized with pressurized oxygen under alkaline conditions at $80,95,110,125$, and $140^{\circ} \mathrm{C}$ for $20,40,60$, and $80 \mathrm{~min}$ using a $1 \mathrm{~L}$ Parr reactor. To avoid metal ion contamination, all interior parts of the reactor (including cooling coils and sampling ports) were coated with Teflon. A customized glass liner was used as the actual reaction flask. The following parameters were applied to all oxidations described in this work: $560 \mathrm{mg}$ of residual kraft lignin was mixed with $400 \mathrm{~mL}$ of deionized, distilled water. Sodium hydroxide $(280 \mathrm{mg}$, which is equivalent to $2.3 \mathrm{wt} . \%$ based on pulp ( $\kappa$ no. 30.9)) was then added, causing solubilization of the lignin. The $\mathrm{pH}$ prior to the onset of the reaction was between 11.5 and 11.6. Prior to placing the lignin sample in the reactor, the whole system was heated to $80^{\circ} \mathrm{C}$. After sealing the system, the reactor was rapidly heated to the desired temperature. It was then purged with oxygen several times prior to being subjected to a constant oxygen pressure (100 psig or $0.68 \mathrm{MPa}$ ) that was maintained for the duration of the oxidation.

To isolate the effect of alkalinity in the absence of oxygen, a series of samples was reacted at $140^{\circ} \mathrm{C}$ using $0.68 \mathrm{MPa}$ of nitrogen pressure under the same conditions described above.

\section{Quantitative recovery of oxidized and fragmented lignins}

After the treatment, the sampling valve of the Parr reactor was opened and an aliquot of lignin solution was cooled to room temperature by passing it through an efficiently cooled reflux condenser. The $\mathrm{pH}$ of the sample was then immediately adjusted to 2 , which caused the remaining polymeric fraction of the lignin to precipitate out. The precipitated lignin was then recovered by centrifugation, washed with deionized distilled water whose $\mathrm{pH}$ had been adjusted to 2, freeze-dried, and stored in a desiccator (see Scheme 4).

The above washings were all combined with the initial filtrate, which contained lower molecular weight lignin fractions combined with hydrophilic lignin fragments. At this point the molecules soluble in organic solvents were extracted with ethyl acetate until the organic layer was no longer coloured. The ethyl acetate extract was allowed to dry in air and stored in a desiccator.

Consequently, the remaining aqueous portion of the sample was enriched in oxidized lignin fragments. The volume of this fraction was first reduced in a rotary evaporator (set at $35-40{ }^{\circ} \mathrm{C}$ ) and it was freeze-dried. The collected solids were then treated with dry dimethylformamide, DMF, filtered to remove inorganic salts, and the filtrate was added dropwise in an excess of diethyl ether $(400 \mathrm{~mL})$, allowing the precipitation of the hydrophilic fragments and washing out excess DMF. Finally, the collected lignin fractions were combined, mixed thoroughly, and stored in a vacuum desiccator prior to ${ }^{31} \mathrm{P}$ NMR analyses.

\section{${ }^{31} \mathrm{P}$ NMR analyses of oxidized lignins}

Quantitative ${ }^{31} \mathrm{P}$ NMR analyses were carried out on the starting and the oxidized lignins using published procedures (18-20). Since oxidized lignins were found to be of limited solubility in pyridine- $\mathrm{CDCl}_{3}$, a new solvent system for the acquisition of these spectra was used in this effort. Approximately $40 \mathrm{mg}$ of our lignin sample was dissolved in $500 \mu \mathrm{L}$ of deuterated DMF. A mixture of pyridine $/ \mathrm{CDCl}_{3}(300 \mathrm{~mL}$, $1.6 / 1 \mathrm{v} / \mathrm{v})$ was then added and the samples were phosphitylated with $100 \mathrm{~mL}$ of 2-chloro-4,4,5,5-tetramethyl-1,3,2dioxaphospholane, synthesized according to published procedures $(21,22)$. Solutions of $100 \mathrm{~mL}$ of cyclohexanol $(1.1 \mathrm{mg})$ and chromium acetylacetonate $(0.55 \mathrm{mg})$ in $10 \mathrm{~mL}$ of pyridine $/ \mathrm{CDCl}_{3}(1.6 / 1 \mathrm{v} / \mathrm{v})$ were also added to serve as the internal standard and relaxation reagent, respectively.

The ${ }^{31} \mathrm{P}$ NMR spectra were acquired on a Varian XL-300 MHz spectrometer (operating at $121.5 \mathrm{MHz}$ ). The chemical shifts reported are relative to the reaction product of water with the phosphitylation reagent, which has been reported to give a sharp signal at $132.2 \mathrm{ppm}(20,23)$. For each spectrum 128 
Table 1. The amounts of the various hydroxyl groups present within the starting and the oxidized residual kraft lignins, determined by quantitative ${ }^{31} \mathrm{P}$ NMR.

\begin{tabular}{|c|c|c|c|c|c|}
\hline $\begin{array}{l}\text { Temperature of } \\
\text { oxidation }\left({ }^{\circ} \mathrm{C}\right)\end{array}$ & $\begin{array}{l}\text { Time of oxidation } \\
(\mathrm{min})\end{array}$ & $\begin{array}{l}\text { Carboxylic acids } \\
(\mathrm{mmol} / \mathrm{g})\end{array}$ & $\begin{array}{l}\text { Guaiacyl phenols } \\
(\mathrm{mmol} / \mathrm{g})\end{array}$ & $\begin{array}{l}\text { Catecholic phenols } \\
(\mathrm{mmol} / \mathrm{g})\end{array}$ & $\begin{array}{l}\text { Aliphatic alcohols } \\
(\mathrm{mmol} / \mathrm{g})\end{array}$ \\
\hline \multirow[t]{4}{*}{80} & 20 & 0.48 & 0.52 & 0.18 & 1.97 \\
\hline & 40 & 0.5 & 0.45 & 0.14 & 1.91 \\
\hline & 60 & 0.51 & 0.43 & 0.16 & 1.78 \\
\hline & 80 & 0.54 & 0.39 & 0.14 & 1.8 \\
\hline \multirow[t]{4}{*}{95} & 20 & 0.41 & 0.57 & 0.19 & 2.07 \\
\hline & 40 & 0.47 & 0.49 & 0.25 & 2.13 \\
\hline & 60 & 0.57 & 0.37 & 0.16 & 1.78 \\
\hline & 80 & 0.56 & 0.34 & 0.26 & 1.76 \\
\hline \multirow[t]{4}{*}{110} & 20 & 0.63 & 0.37 & 0.16 & 1.72 \\
\hline & 40 & 0.71 & 0.26 & 0.15 & 1.65 \\
\hline & 60 & 0.73 & 0.19 & 0.13 & 1.68 \\
\hline & 80 & 0.92 & 0.19 & 0.18 & 1.48 \\
\hline \multirow[t]{4}{*}{125} & 20 & 0.62 & 0.38 & 0.24 & 1.74 \\
\hline & 40 & 1.14 & 0.25 & 0.22 & 1.71 \\
\hline & 60 & 1.16 & 0.24 & 0.22 & 1.89 \\
\hline & 80 & 1.21 & 0.21 & 0.21 & 1.81 \\
\hline \multirow[t]{4}{*}{140} & 20 & 0.98 & 0.21 & 0.27 & 1.33 \\
\hline & 40 & 1.1 & 0.2 & 0.18 & 1.42 \\
\hline & 60 & 1.4 & 0.15 & 0.23 & 1.23 \\
\hline & 80 & 1.5 & 0.11 & 0.17 & 1.13 \\
\hline Control lignin & & 0.26 & 0.57 & 0.2 & 2.01 \\
\hline
\end{tabular}

Fig. 1. Typical quantitative ${ }^{31} \mathrm{P}$ NMR spectra, signal assignment, and integration ranges applied during the course of this work. The phosphitylation reagent employed was 2 -chloro-4,4,5,5tetramethyl-1,3,2-dioxaphospholane $(19,20)$.

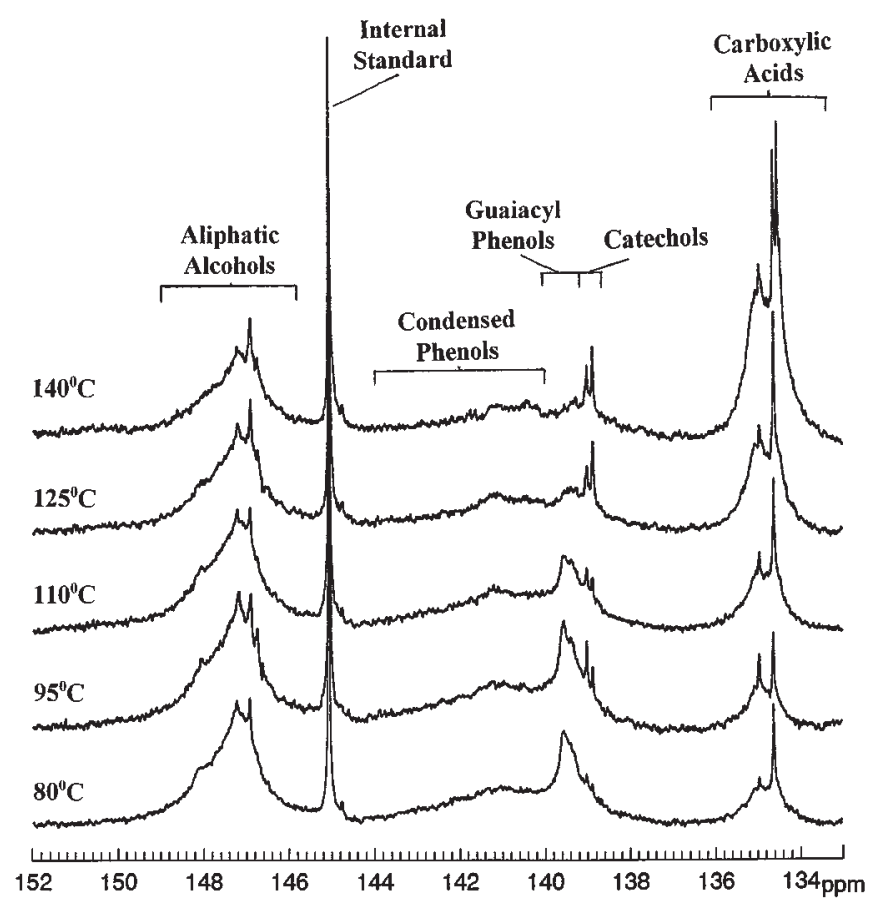

transients were acquired with a delay time of $25 \mathrm{~s}$ between acquisitions.

\section{Results and discussion}

The oxidation of residual kraft lignin under high pressure and alkalinity is known to partially degrade the lignin with concomitant formation of functional groups such as quinones and carboxylic acids (24), significantly altering its solubility. Since the primary objective of this work was to identify and quantify these structural changes under conditions of an oxygen delignification stage, extensive efforts were made to quantitatively isolate the lignin after the reaction.This ensured that most of the highly oxidized and low molecular weight fragments were included in the quantitative ${ }^{31} \mathrm{P}$ NMR analyses that followed. To accomplish this, a multistep procedure was developed (Scheme 4) to recover all of the species possessing different solubility characteristics in different solvents.The application of this scheme provided recovery yields between $75-95 \%$.

Careful optimization work showed that the oxidized lignins could be divided into three distinct fractions. One fraction could easily be precipitated out of aqueous alkali by decreasing its $\mathrm{pH}$ to 2 . More hydrophilic fractions were then recovered by extracting aqueous lignin solutions with ethyl acetate. Finally, highly oxidized lignin macromolecules and fragments were isolated by selectively dissolving them with dimethylformamide (DMF), separating them from inorganic salts. It should be noted that no attempt was made to recover 
Scheme 5. The major reactions involving phenolic units and free radical species during oxygen delignification (9-16).

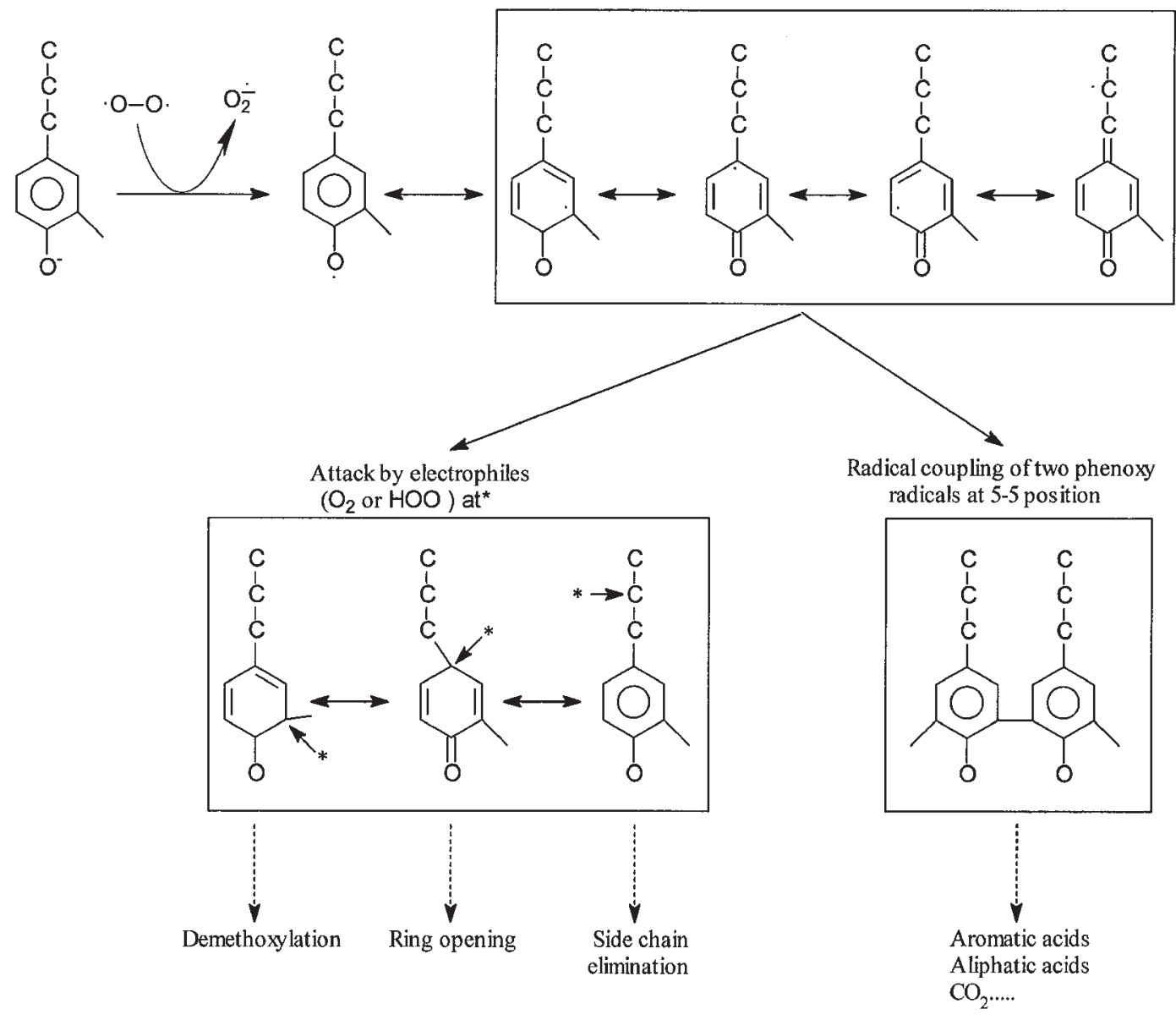

and quantify volatile by-products such as $\mathrm{CO}, \mathrm{CO}_{2}$, and methanol.

A series of typical quantitative ${ }^{31} \mathrm{P}$ NMR spectra of softwood residual kraft lignins obtained after phosphitylation with 2-chloro-4,4,5,5-tetramethyl-1,3,2-dioxaphospholane is displayed in Fig. 1. The integration ranges and signal assignment were similar to those reported by Sun and Argyropoulos $(23,25)$ based on previous model compound (20) and lignin studies (19). Therefore, the foundations of the following discussion belong in the quantitative ${ }^{31} \mathrm{P}$ NMR data of Table 1, which summarizes the amounts of the various hydroxyl groups present within the starting and oxidized residual kraft lignins.

\section{The reactivity of guaiacyl phenolic units with oxygen}

Molecular oxygen, in alkaline media at elevated temperatures, has been considered to behave as a biradical species $(9,13)$. Therefore, the first step in the oxidative degradation of phenolic units is thought to be the abstraction of an electron from the phenolate anion by biradical oxygen, giving the corresponding phenoxy and superoxide anion radicals (9). At the same time, the protonation of reduced oxygen gives rise to hydroperoxy radicals, which are known to behave as stronger oxidants than molecular oxygen (13), reacting faster with lignin units. As such, phenoxy radicals are attacked by hydroperoxy radicals at the centres of high electron densities as shown in Scheme 3. The reaction products are known to be the relatively unstable cyclohexadienone hydroperoxide intermediates (14-16), leading to demethoxylation, ring-opening, and side-chain elimination reactions (Schemes 5 and 6). In fact, such species have been detected, isolated, and quantified as photochemical transformation products of commercial antioxidants (26-28). The demethylation and ring-opening reactions give rise to catechols and various mono and dicarboxylic acids, such as maleic and muconic acids (Scheme 6), while the side-chain elimination reactions cause the formation of other organic acids and para-quinones, thus depolymerizing lignin. The outcome of all these reactions is the actual elimination of guaiacyl phenolic units.

In an attempt to shed more light in this complex series of events, the profiles of guaiacyl phenolic group elimination during the oxidation of residual kraft lignin were determined and are plotted as a function of temperature and time in Fig. 2.

It is apparent that the rate of guaiacyl phenolic unit elimination increases as the oxidation intensifies. The reaction temperature, rather than the reaction time, seems to be of primary significance in causing the elimination of these units. The rate of guaiacyl unit elimination is considerably slower at 80 and $95^{\circ} \mathrm{C}$ than at 110,125 , or $140^{\circ} \mathrm{C}$. Furthermore, the degradation of guaiacyl units at temperatures above $100^{\circ} \mathrm{C}$ seems to proceed in two distinct phases: an initial rapid decrease of the guaiacyl units is observed within 20-40 min, followed by a significantly slower regime. Similarly, Lai et al. (29) claimed that $10 \%$ of these uncondensed phenolic 
Scheme 6. Reactions proposed to occur under oxygen delignification conditions involving phenolic moieties and superoxide anion radicals via dioxetane intermediates (14).<smiles>CCCC1C=CC(=O)C(OC)=C1</smiles><smiles>CCC1=CC(OC)C(=O)C=C1</smiles><smiles>CCCC12C=C(OC)C(CCCC1)C(O)CCCO2</smiles><smiles>CCCC1(O)C=C(OC)C(=O)C2OC21</smiles><smiles>COC(=O)C=C(/C=C\C(=O)O)CCCCN</smiles><smiles>COC1C=C(C(C)=O)C=CC1O</smiles>

units are eliminated rapidly during oxygen delignification at $100^{\circ} \mathrm{C}$ and 90 psi oxygen pressure $(1 \mathrm{psi}=6.9 \mathrm{kPa})$, followed by a slow phase during which their concentrations remain relatively constant. Such behaviour is reminiscent of the kappa number reduction profiles usually encountered during oxygen delignification of kraft pulps. Physical constraints such as a diffusion-controlled reaction whose rate depends on the rate of transport of reagents from one phase to another could be responsible for stalling the reactivity in the slow phase (30). However, the data of Fig. 2 represent the oxidative elimination of guaiacyl phenolic units from within residual kraft lignin under homogeneous oxygen delignification conditions, making it likely that guaiacyl phenolic units may exist within residual kraft lignin whose reactivity toward oxidative reactions is different. Branching and crosslinking variations that occur within lignin may be responsible for the observed reactivity and (or) accessibility variations.
Fig. 2. Three-dimensional plots depicting the oxidative elimination of guaiacyl phenolic units from within residual kraft lignin under homogeneous oxygen delignification conditions.

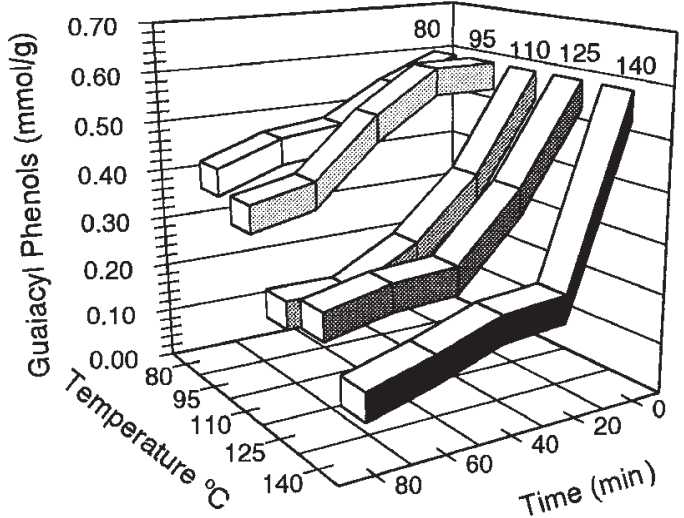

Fig. 3. Three-dimensional plots depicting the buildup of carboxylic acids within residual kraft lignin as a function of time and temperature under homogeneous oxygen delignification conditions.

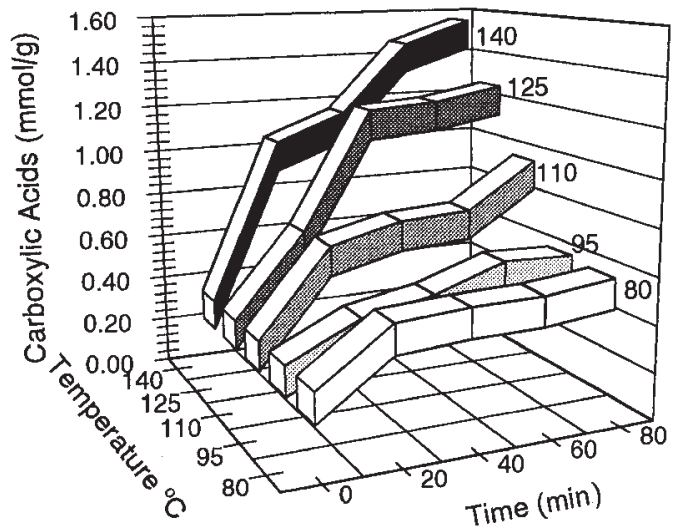

Formation of carboxylic acids within residual kraft lignin

The presence of carboxylic acid groups on the lignin macromolecule is considered to promote lignin solubilization in alkaline media during oxygen delignification $(10,23)$ or other bleaching stages (25). Lignin oxidation reactions may induce the formation of carboxylic acids via a number of pathways either as monomeric fragments or functional groups attached at the end of side chains of the lignin macromolecules (12, 31,32 ). The most significant pathway involves side-chain elimination and ring-opening reactions in phenolic centres. Side-chain elimination reactions would form vanillin-like structures (which degrade further) while ring-opening reactions lead to moieties resembling muconic acid moieties (16). Another pathway may involve an electrophilic attack at lignin centres of high electron density, causing the formation of a four- membered cyclic peroxide intermediate termed dioxetane $(14,16,24)$; (Scheme 6). This reactive species rearranges to induce efficient cleavage of $\mathrm{C}-\mathrm{C}$ bonds. It should be noted that after the initial electrophilic attack, the molecule may also be attacked by external nucleophiles, but according to Gierer (14) these are only minor reactions. 
Scheme 7. Possible reactions, operating on phenolic units, that may account for the formation and subsequent elimination of catechols, during the oxidation of residual kraft lignin at elevated temperatures and alkalinities.

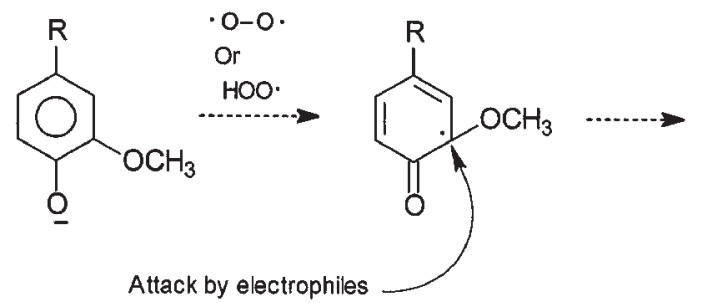

Fig. 4. Three-dimensional plots depicting the $\mathrm{pH}$ profiles of the medium as a function of time and temperature during the oxidation of residual kraft lignin, under homogeneous oxygen delignification conditions.

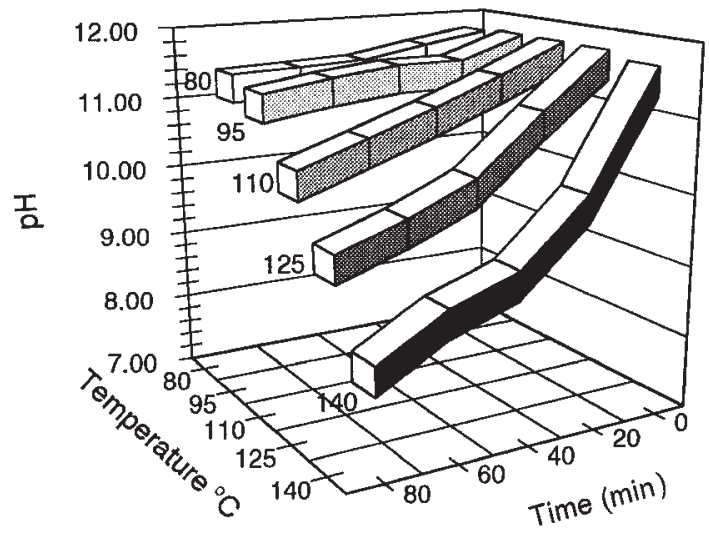

Figure 3, displaying the three-dimensional plots for the buildup of carboxylic acids within residual kraft lignin as a function of time and temperature, is very revealing. For all temperatures studied, two regimes can be distinguished describing the rate of carboxylic acid group formation: an initial rapid phase that dominates the process for the first $20 \mathrm{~min}$ (at all temperatures), followed by a slower one. At temperatures between 125 and $140^{\circ} \mathrm{C}$, the rate of carboxylic acid group formation within the first $20 \mathrm{~min}$ of the process is very high. For example, the concentration of these groups after $20 \mathrm{~min}$ of reaction reaches and exceeds $1.0 \mathrm{mmol} / \mathrm{g}$; while at $80-95^{\circ} \mathrm{C}, 80 \mathrm{~min}$ of reaction caused only a minor enrichment of the lignin structure in carboxylic acid groups (less than $0.5 \mathrm{mmol} / \mathrm{g}$ ).

The rate of carboxylic acid group formation seems to dramatically increase as the reaction temperature increases, analogous to the profiles obtained for the elimination of guaiacyl phenolic units. It is interesting to observe that only minor oxidation occurs within the residual kraft lignin at reaction temperatures typical of conventional commercial oxygen delignification installations $\left(80-100^{\circ} \mathrm{C}\right)$.

At these temperatures, increasing the reaction time does not seem to significantly aid the formation of carboxylic acid groups. Higher temperatures, however, cause the concentration of carboxylic acid groups to steadily increase as a function of treatment time. In general, the efficiency of oxidation of residual kraft lignin significantly increases above $100^{\circ} \mathrm{C}$ even though these experiments were conducted under idealized two-phase homogeneous conditions. The data imply that a three-phase oxygen delignification system operat-<smiles>[R]C1=CC(=O)C(=O)C(CCCC)C=C1</smiles>

ing at temperatures below $100^{\circ} \mathrm{C}$ causes only minor oxidative changes on the structure of the lignin on the fibre.

\section{The effect of oxidation on the $\mathrm{pH}$ of the medium}

Since $\mathrm{pH}$ is known to seriously affect the efficiency of an oxygen delignification stage, it was deemed appropriate to examine the dependence of $\mathrm{pH}$ as a function of temperature and time during the oxidation of residual kraft lignin. The amount of sodium hydroxide used in the present sequence of experiments was similar to that used during a commercial softwood oxygen delignification installation $(2.3 \%$ based on oven dry fibre, see Experimental).

The data of Fig. 4 show the contribution of the oxidized groups in residual kraft lignin toward lowering the $\mathrm{pH}$ of the aqueous medium during oxygen delignification conditions. In a manner analogous to the formation of carboxylic acid groups (Fig. 3), the $\mathrm{pH}$ of the medium is practically unaffected at temperatures below $100^{\circ} \mathrm{C}$. This could be significant information when one considers the design of oxygen delignification operations at temperatures above $100^{\circ} \mathrm{C}$. For such a case, one should make provisions so that enough alkali is present in the medium to compensate for the greater $\mathrm{pH}$ losses that the oxidation of lignin will cause above $100^{\circ} \mathrm{C}$. A benchmark observation guiding such a design could be that at $110^{\circ} \mathrm{C}$ and after $80 \mathrm{~min}$ of reaction, the $\mathrm{pH}$ of the medium was found to be slightly below 10 . The trend of decreasing $\mathrm{pH}$ in an alkaline oxidative extraction was observed previously when the temperature was raised from 70 to $110^{\circ} \mathrm{C}$, with some benefits observed by increasing the alkali charge (33).

Another interesting feature of the data of Fig. 4 is the fact that the $\mathrm{pH}$ profiles show two- phase reactions only at temperatures of 125 and $140^{\circ} \mathrm{C}$, in contrast to the data of Fig. 3 (see relevant discussion). At lower temperatures the $\mathrm{pH}$ decrease is smooth throughout the examined time range.

\section{Oxidative demethoxylation reactions}

Model compound studies have shown that demethoxylation reactions may occur under oxygen delignification conditions (13). However, there has been no direct quantitative evidence for the occurrence of such reactions in residual kraft lignin during oxygen delignification. Despite the fact that the exact mechanism of demethoxylation is not known, Kratzl et al. (13) have assumed that a phenoxy radical may react with oxygen or hydroperoxy radicals, yielding cyclohexadienone hydroperoxides (Scheme 7). For these species, after demethoxylation, rearrangement and ring rearomatization yield catechols that are easily oxidized to ortho quinones and then to a muconic acid type of structures (13). 
Fig. 5. Three-dimensional plots depicting the profile of catechol formation as a function of time and temperature during the oxidation of residual kraft lignin under homogeneous oxygen delignification conditions.

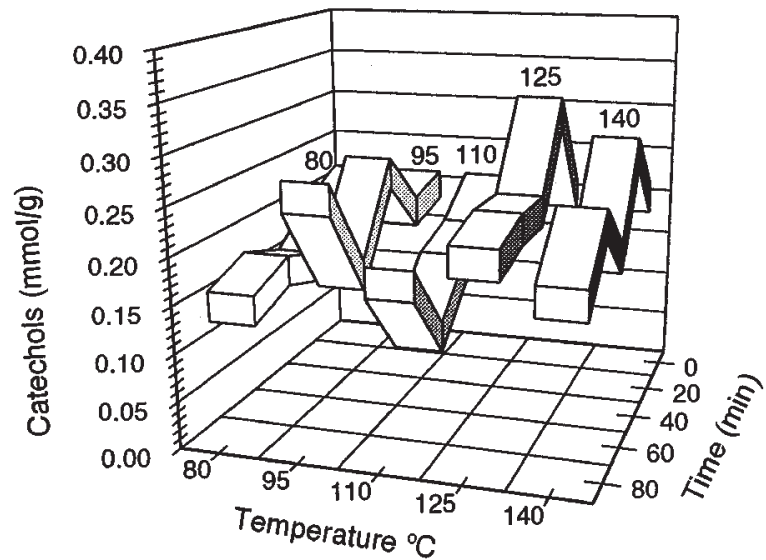

Fig. 6. A comparison of carboxylic acid group formation on residual kraft lignin in the presence and absence of oxygen at $140^{\circ} \mathrm{C}$.

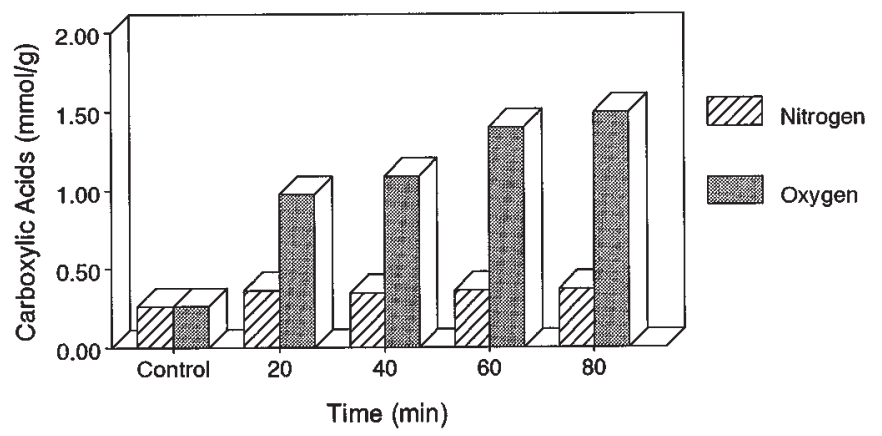

The quantitative ${ }^{31} \mathrm{P}$ NMR spectra of Fig. 1 show the characteristic signals of catechols in the region 138.6-139.1 ppm $(19,20,23)$. Apparently, catechols form during the oxidation of residual kraft lignin, since these signals are not as obvious in the spectrum of the starting material (Fig. 1). Since this novel methodology allows the detection and quantification of catechols in a lignin sample, it was used to quantify them as a function of time and temperature (Fig. 5).

One can observe some minor but irregular variations in the abundance of catechols within oxidized residual kraft lignin as a function of time and temperature (Fig. 5). In general, their concentration can be considered relatively constant over the whole temperature range examined, implying that catechols are involved in the oxidation process as intermediates. Their participation in an oxygen delignification has been suggested by Gellerstedt and Lindfors (30) and Renard et al. $(31,32)$. It is likely that while there is a supply of guaiacyl units in the system, these intermediates would form and then be oxidized in a continuous fashion. In addition to the catechols acting as important intermediates in the oxidation process, they also increase the hydrophilicity of the lignin, augmenting its solubility in alkaline media.
Fig. 7. A comparison of condensed phenolic units on residual kraft lignin in the presence and absence of oxygen at $140^{\circ} \mathrm{C}$.

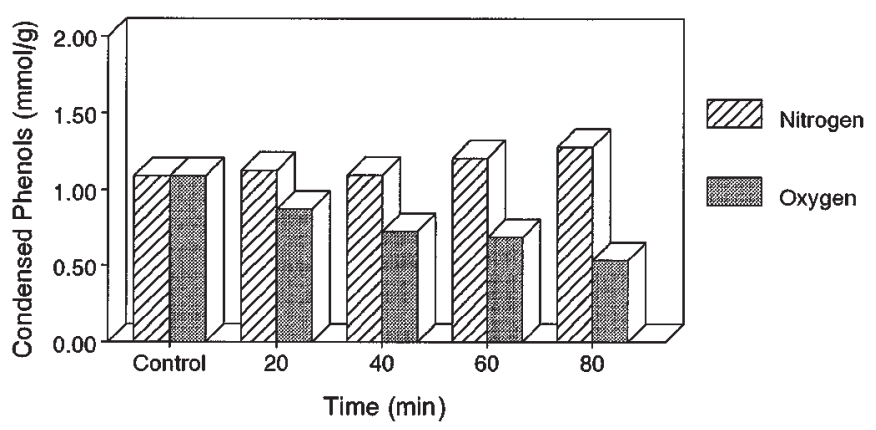

\section{The effect of alkali and temperature on the reaction pathways}

During this effort we attempted to isolate the effect of alkali and temperature on the structure of residual kraft lignin by substituting pressurized oxygen with nitrogen, in otherwise identical conditions to those described so far. To emphasize the overall effects, this study was conducted at $140^{\circ} \mathrm{C}$ and the functional group distribution changes induced on the residual kraft lignin were compared to those in Figs. 2 and 4 obtained at $140^{\circ} \mathrm{C}$.

The dramatic effect of oxygen toward inducing the formation of carboxylic acid groups within residual kraft lignin, under oxygen delignification conditions, is shown in Fig. 6. In the absence of oxygen, temperature $\left(140^{\circ} \mathrm{C}\right)$ and alkali alone do not induce the formation of carboxylic acids in residual kraft lignin. For comparison purposes under the same conditions and in the presence of oxygen, an approximate $450 \%$ increase in carboxylic acid content was measured.

Phenolic structures that are substituted at the C5 position may be termed condensed phenols. The fate of such units, under both an oxidative and an inert atmosphere, at $140^{\circ} \mathrm{C}$, is shown in Fig. 7. It appears that at $140^{\circ} \mathrm{C}$ the amount of condensed phenolic units decreased over $50 \%$ via oxidative degradation reactions. However, in the absence of oxygen, the condensed phenolic units increased by about $17 \%$. It is therefore likely that this increase describes alkaline condensation reactions taking place within residual kraft lignin under the examined conditions. At elevated alkalinities and temperatures, phenoxy anions (33) may attack the $\alpha$ position of other $\mathrm{C} 9$ units and consequently, after rearrangement, new $\mathrm{C}_{\alpha}-\mathrm{C}_{5}$ units may form, see Scheme $8(34,35)$.

In addition, any residual $\beta-\mathrm{O} 4$ units present in residual kraft lignin, albeit in minor amounts (36), may rearrange and cause the formation of minor amounts of formaldehyde. These species may then methylolate the $\mathrm{C} 5$ position of phenolic units, eventually creating new diphenyl methane structures (Scheme 8).The data of Fig. 7, therefore, reveal a concealed set of condensation reactions.

Figure 8 displays the changes in the actual amounts of guaiacyl phenolic units under inert and oxidative conditions. In the presence of oxygen, the amount of guaiacyl phenolic units decreases but in the absence of oxygen, the amount of guaiacyl phenolic units increased somewhat. The rationale that may be offered to explain these data is that under the examined conditions, the small amounts of $\beta-\mathrm{O} 4$ bonds that have been documented to remain on residual kraft lignin 
Scheme 8. Alkaline condensation reactions that may operate on phenolic units of residual kraft lignin (34).

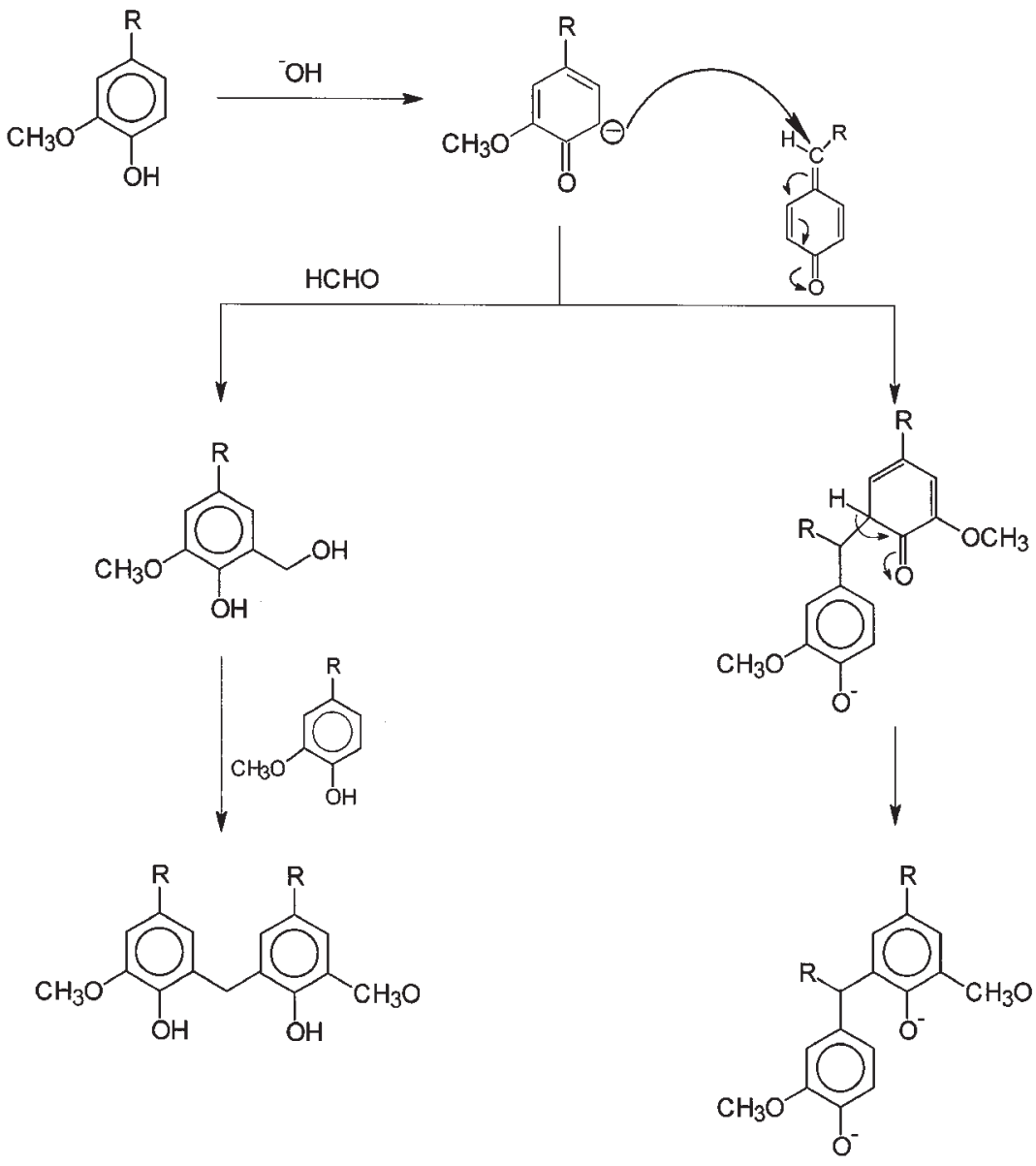

Fig. 8. A comparison of the fate of guaiacyl phenolic units present on residual kraft lignin in the presence and absence of oxygen at $140^{\circ} \mathrm{C}$.

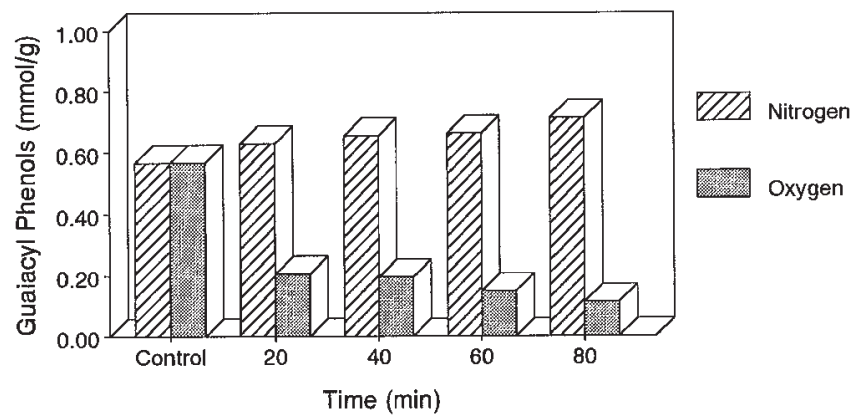

may cleave (36), and will inevitably cause the formation of new guaiacyl phenolic units.

\section{Concluding remarks}

During oxygen delignification the guaiacyl phenolic units of softwood residual kraft lignin eliminate with the concomitant formation of carboxylic acids. The rates for the elimination of phenols and the formation of acids were found to significantly increase when the reaction temperature was above $100^{\circ} \mathrm{C}$. The concentration of catechols was found to be relatively constant over the whole temperature range ex- amined, implying that catechols are involved in the oxidation process as intermediates.

In general, reaction temperatures varying between 80 and $100^{\circ} \mathrm{C}$ caused only minor functional group changes on softwood residual kraft lignin. The reactivity of this kraft lignin substantially increased at temperatures above $110^{\circ} \mathrm{C}$. Therefore considerable oxidative delignification should be obtained at elevated temperatures. The challenge remains, however, for developing strategies that may prevent the unwanted deterioration of pulp quality.

\section{Acknowledgements}

The authors would like to thank Ms. B. Van Lierop and Mr. Y. Sun for their input while reviewing the manuscript. The constructive criticism of Dr. Sten Ljunggren of the Swedish Pulp and Paper Research Institute is also gratefully acknowledged. This research was supported by the Goverment of Canada in the form of a Strategic Grant and by the Air Products \& Chemicals Corporation.

\section{References}

1. T.J. McDonough. TAPPI, 69(6), 46 (1986).

2. V. Masura. Cellul. Chem. Technol. 27, 201 (1993).

3. L. Olm and A. Teder. TAPPI, 62(12), 43 (1979).

4. D. Lachenal and C. DeChoudens. Cellul. Chem. Technol. 20, 553 (1986). 
5. C.L. Hsu and J.S. Hsieh. TAPPI, 70(12), 197 (1987).

6. S. Ljunggren. Nord. Pulp Paper Res. J. 1, 38, (1990).

7. S. Ljunggren and E. Johansson. Nord. Pulp Paper Res. J. 5(3), 148 (1990).

8. S. Ljunggren. J. Pulp Paper Sci. 12(2), 54 (1986).

9. K. Kratzl, J. Gratzl, and P. Claus. Adv. Chem. Ser. 59, 157 (1966).

10. G. Gellerstedt, K. Gustafsson, and E.-L. Lindfors. Nord. Pulp Paper Res. J. 1(3), 14 (1986).

11. E. Johansson and S. Ljunggren. J. Wood Chem. Technol. 14(4), 507 (1994).

12. S. Ljunggren, G. Gellerstedt, and M. Petterson. 6th Int. Symp. Wood and Pulping Chem. 1, 229 (1991).

13. K. Kratzl, P. Claus, W. Lonsky, and J. Gratzl. J. Wood Sci. Technol. 8, 35 (1974).

14. J. Gierer. Int. Symp. Wood and Pulp Chemistry, Paris. 1987. p. 279.

15. J. Gierer. Proc. Proc. Int. Symp. Wood and Pulping Chemistry CTAPI, Beijing. 1993. p. 303.

16. J. Gierer and F. Imsgard. Sven. Papperstidn. 16, 510 (1977).

17. Z. Jiang and D. S. Argyropoulos. J. Pulp Pap. Sci. 20(7), J183 (1994).

18. D.S. Argyropoulos. J. Wood Chem. Technol. 14, 45 (1994).

19. A. Granata and D.S. Argyropoulos. J. Agric. Food Chem. 43, 1538 (1995).

20. Z.-H. Jiang, D.S. Argyropoulos, and A. Granata. Magn. Reson. Chem. 33, 375 (1995).

21. A. Zwierzak. Can. J. Chem. 45, 2501 (1967).

22. H.J. Lucas, F.W. Mitchell, Jr., and C.N. Scully. J. Am. Chem. Soc. 72, 4591 (1950).
23. Y. Sun and D.S. Argyropoulos. J. Pulp Paper Sci. 21(6), J185 (1995).

24. H.-M. Chang and J. Gratzl. Proc. Int. Symp. Chemistry of Delignification with Oxygen, Ozone, and Peroxides: Ring Cleavage Reaction of Lignin Models with Oxygen and Alkali. 1986. p. 151.

25. Y. Sun and D.S. Argyropoulos. Holzforschung, 50(2),175 (1996).

26. T. Matsuura, K. Omura, and R. Nakashima. Bull. Chem. Soc. Jpn. 38, 1398 (1965).

27. L. Taimr and J. Pospisil. Makromol. Chem. 39, 189 (1974); 39, 619 (1974).

28. R.G. Lichtenthaler and F. J. Ranfelt. 148, 553 (1978).

29. Y.-Z. Lai, M. Funaoka, and H.-T. Chen. Holzforschung, 48(4), 355 (1994).

30. G. Gellerstedt and E. -L. Lindfors. Tappi J. 70, 119 (1987).

31. J.J. Renard, D.M. Mackie, and H.I. Bolker. Pap. Puu, 57(11), 1 (1975).

32. J.J. Renard, D.M. Mackie, H.I. Bolker, and D.W. Clayton. Cellul. Chem. Technol. 9, 341 (1975).

33. W.I. Taylor and A.R. Battersby. In Oxidative coupling of phenols. Chap. 1: Phenol coupling. Marcel Dekker, Inc., New York. 1967.

34. J. Marton. Reactions in alkaline pulping. In Lignins: occurrence, formation, structure, and reactions. John Wiley and Sons, Inc., New York. 1971. pp. 639-694.

35. J. Gierer. Wood Sci. Technol. 14, 241 (1980).

36. M. Kleen, B. Hortling, and T. Tamminen. 4th Eur. Workshop on Lignocellulosics and Pulp, Stresa, Italy, Sept. 8-11, 1996. pp. $44-50$. 